Meta

Journal des traducteurs

Translators' Journal

\title{
La pluridimensionnalité du concept. Approche terminologique du domaine de la spatiologie
}

\section{Rima Baraké}

Volume 53, numéro 4, décembre 2008

URI : https://id.erudit.org/iderudit/019655ar

DOI : https://doi.org/10.7202/019655ar

Aller au sommaire du numéro

Éditeur(s)

Les Presses de l'Université de Montréal

ISSN

0026-0452 (imprimé)

1492-1421 (numérique)

Découvrir la revue

Citer cet article

Baraké, R. (2008). La pluridimensionnalité du concept. Approche terminologique du domaine de la spatiologie. Meta, 53(4), 907-915. https://doi.org/10.7202/019655ar
Résumé de l'article

À partir de l'analyse de plusieurs termes se rapportant au domaine de la spatiologie, dans des langues aussi différentes que l'anglais et le français d'une part et l'arabe d'autre part, nous nous proposons de démontrer l'importance de la notion de pluridimensionnalité du concept dans l'organisation des connaissances et la classification des objets du monde. Ce faisant, nous aboutirons aussi à la conclusion que la structuration d'un domaine de spécialité, l'élaboration de son arborescence et surtout la formulation d'une définition dépendent principalement des caractères pris en compte dans l'appréhension des concepts, donc nécessairement de la " dimension » du concept. 


\title{
La pluridimensionnalité du concept. Approche terminologique du domaine de la spatiologie
}

\author{
RIMA BARAKÉ \\ Université de la Sorbonne Nouvelle, Paris, France \\ barake_rima@hotmail.com
}

\begin{abstract}
RÉSUMÉ
À partir de l'analyse de plusieurs termes se rapportant au domaine de la spatiologie, dans des langues aussi différentes que l'anglais et le français d'une part et l'arabe d'autre part, nous nous proposons de démontrer l'importance de la notion de pluridimensionnalité du concept dans l'organisation des connaissances et la classification des objets du monde. Ce faisant, nous aboutirons aussi à la conclusion que la structuration d'un domaine de spécialité, l'élaboration de son arborescence et surtout la formulation d'une définition dépendent principalement des caractères pris en compte dans l'appréhension des concepts, donc nécessairement de la «dimension» du concept.
\end{abstract}

\begin{abstract}
After analyzing several terms related to space science, in languages as different as French and English on one hand and Arabic on the other hand, we intend to demonstrate the importance of the concept's multidimensionality in the organization of knowledge and the classification of objects. This will lead us to the conclusion that the structure of a field, the elaboration of its tree diagram and mostly the formulation of a definition depend mainly on the characteristics taken into account in the apprehension of concepts, and therefore on the "dimension" of the concept.
\end{abstract}

MOTS-CLÉS/KEYWORDS

dimension, concept, caractère, spatiologie, organisation des connaissances

Nous savons que le concept est l'unité de base de toute analyse terminologique. Que celle-ci soit synchronique ou diachronique, portant sur le terme ou sur la définition, il faut toujours revenir au concept, à sa description au sein du système de concepts qu'il constitue avec les autres concepts appartenant au même domaine.

Le concept est une «unité de connaissance créée par une combinaison unique de caractères" (ISO 1087-1 2000 : 2). Cette définition que donne la norme ISO 108712000 du concept met surtout l'accent sur la décomposition du concept en caractères, une décomposition qui permet une meilleure compréhension du concept et donc une meilleure organisation du système de concepts auquel il appartient. 


\section{Concept et caractères}

Si le concept est, selon cette définition, une unité de connaissance, cela ne veut pas dire qu'il est une unité fixe, bien au contraire. En fait, il évolue avec les progrès scientifiques et techniques; il se précise avec l'affinement des connaissances, ce qui entraîne nécessairement un changement des caractères qui le composent. Le caractère, qui est la «propriété abstraite d'un objet, ou d'un ensemble d'objets " (ISO 108712000 : 3) - le terme «objet» étant pris ici au sens terminologique, c'est-à-dire «tout ce qui peut être perçu ou conçu» (ISO 1087-1 2000: 2) -, est l'unité constituante du concept et reflète donc une connaissance de l'objet, de toutes ses propriétés, ses fonctions, etc. Par conséquent, toute nouvelle découverte ou connaissance plus approfondie de l'objet entraînera nécessairement une précision et une clarification du concept qui lui correspond.

Les terminologues sont d'accord sur le fait que plus un concept est précis, c'està-dire doué de plus de caractères, plus il est facile de le distinguer des concepts voisins, appartenant au même domaine.

On peut donner à titre d'exemple le concept //planète// [les concepts seront notés entre // //] qui est resté, jusqu'en août 2006 - date de la $2^{\mathrm{e}}$ Assemblée générale de l'Union astronomique internationale (ou UAI) durant laquelle la dernière définition de //planète// a été votée -, un concept instable, ayant des frontières floues avec les concepts voisins.

À partir de trois définitions prises à des époques différentes, nous avons pu tirer les caractères qui composent ou composaient le concept //planète// :

1. //étoile errante////mouvement réglé et périodique// (Furetière 1690)

2. //corps céleste////révolution autour du Soleil////change continuellement de position par rapport aux autres étoiles// (Diderot et d'Alembert 1750)

3. //corps céleste// //en orbite autour du Soleil////masse suffisante pour que sa gravité l'emporte sur les forces de cohésion du corps solide et le maintienne en équilibre hydrostatique// //a éliminé tout corps se déplaçant sur une orbite proche// (UAI, août 2006)

On peut ainsi remarquer qu'en 1690 le concept //planète// faisait partie du concept générique //étoile// qui se divisait en deux concepts spécifiques: //étoile errante// et //étoile fixe//.

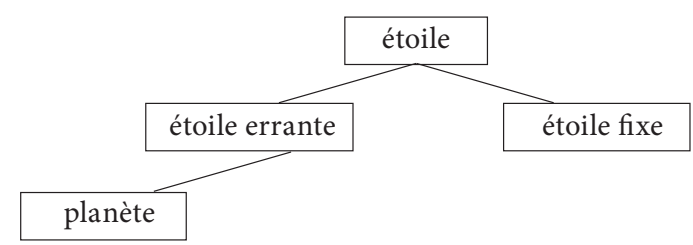

En 1750 est apparu un nouveau concept, //corps céleste//, qui englobait les deux concepts //étoile// et //planète//. La distinction entre ces deux concepts se faisait à l'aide du caractère //révolution autour du Soleil//, caractéristique de //planète//. Cependant, la frontière reste floue entre //planète// et //objet céleste//, les caractères formant le premier concept pouvant également s'appliquer à la majorité des concepts spécifiques de //objet céleste//, et en particulier au concept //astéroïde//. 


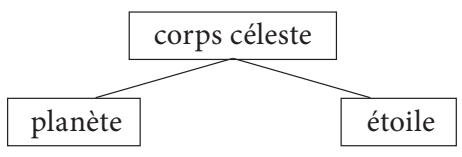

Ce n'est qu'en 2006 que //planète// s'est stabilisé et délimité par rapport aux concepts voisins, et cela grâce à un processus de développement et de spécification des caractères qui le constituent:

a. //corps céleste//;

b. //en orbite autour du Soleil//;

c. //possède une masse suffisante pour que sa gravité l'emporte sur les forces de cohésion du corps solide et le maintienne en équilibre hydrostatique//;

d. //a éliminé tout corps se déplaçant sur une orbite proche//.

La décomposition du concept en caractères est donc importante pour délimiter celuici par rapport aux autres concepts contigus, pour définir et établir les relations qu'ils entretiennent entre eux et ensuite, à une échelle plus grande, pour déterminer la structure du système de concepts du domaine.

Cependant, l'utilité de l'analyse des caractères ne se limite pas à la compréhension et la délimitation du concept, puisqu'ils entrent dans l'élaboration de la définition du concept, et parfois dans la formation de la désignation.

En effet, un terme rend parfois compte de certains caractères du concept. Inversement, les caractères composant un concept peuvent servir à la formation du terme désignant ce concept (caractères soulignés dans l'exemple):

//étoile polaire//: //étoile///visible// //indiquant le pôle nord céleste//

Dans cette perspective, Thoiron parle d' "éléments de nomination »: «De même qu'un concept est divisible en traits conceptuels, on peut considérer qu'un terme est constitué d'éléments de nomination, eux-mêmes composés de un ou plusieurs morphèmes » (Thoiron et al. 1996).

Dans l'exemple «étoile polaire», les deux éléments de nomination seraient «étoile» et «polaire» et seraient respectivement en relation avec les caractères (ce que Thoiron nomme «traits conceptuels») //étoile // et //indiquant le pôle nord céleste//.

Depecker affirme que la définition terminologique «peut être considérée comme un microsystème constitué par l'énoncé des caractères du concept et des relations qu'ils forment entre eux» (Depecker 2003: 68). Elle doit donc, par sa nature même, mettre en évidence les caractères qui composent le concept et qui le différencient nettement des autres concepts.

Prenons les définitions des concepts //planète//, //planète naine//, //satellite// et //astéroïde// :

1. //planète//: corps céleste qui est en orbite autour du Soleil, qui possède une masse suffisante pour que sa gravité l'emporte sur les forces de cohésion du corps solide et le maintienne en équilibre hydrostatique (forme sphérique), et qui a éliminé tout corps se déplaçant sur une orbite proche.

2. //planète naine//: corps céleste qui est en orbite autour du Soleil, qui possède une masse suffisante pour que sa gravité l'emporte sur les forces de cohésion du corps solide et le maintienne en équilibre hydrostatique (sous une forme presque sphérique), qui n’est pas un satellite, mais qui n'a pas fait place nette dans son voisinage orbital. 
3. //satellite//: corps céleste qui gravite autour d'un autre dont la masse est supérieure.

4. //astéroïde//: corps céleste dont la taille varie de quelques dizaines de mètres à plusieurs kilomètres de diamètre et qui tourne autour du Soleil sur une orbite faiblement elliptique.

En comparant les caractères que contient chaque définition (marqués par un trait), on remarque les faits suivants:

- les quatre concepts ont pour concept générique //corps céleste//;

- la distinction entre //planète// et //planète naine// se fait essentiellement grâce aux caractères distinctifs suivants: //a éliminé tout corps se déplaçant sur une orbite proche// //n'a pas fait place nette dans son voisinage orbital//;

- le caractère //gravite autour d'un autre dont la masse est supérieure// permet de distinguer // satellite// des autres concepts;

- le caractère distinctif de //astéroïde// est //taille varie de quelques dizaines de mètres à plusieurs kilomètres de diamètre//; etc.

Les caractères relevés dans les définitions ci-dessus ne sont pas les seuls caractères qui forment ces concepts. En effet, tous les caractères conceptuels n'ont pas la même importance; par la suite, tous les caractères n'entrent pas dans la formation de la définition ou du terme. Un choix doit être fait. Il existe un grand nombre de critères qui conditionnent ce choix et font qu'on opte pour tels caractères et non pas pour tels autres. Comme l'affirme Depecker, «on ne choisit pas au hasard les critères de sélection des caractères, mais [...] on le fait dans une certaine optique» (Depecker 2003: 86).

Parmi ces critères, on peut citer:

- le domaine d'usage ou d'application: un grand nombre de sciences entrent dans l'étude des objets et phénomènes spatiaux: la chimie (ou astrochimie), la physique (ou astrophysique), la photographie (ou astrophotographie), la médecine (ou médecine spatiale), la sismologie (ou astrosismologie), la biologie (ou exobiologie), etc. Chacune de ces sciences aborde un aspect ou une propriété des objets et phénomènes spatiaux, et par conséquent chaque science, au sein même de la spatiologie, fait un choix de caractères différents les uns des autres;

- le point de vue;

- l'utilisateur: Le Guern, dans son ouvrage Les deux logiques du langage, donne l'exemple du botaniste et du cuisinier: "Le botaniste et le cuisinier parlent bien la même langue, mais leurs univers de discours diffèrent» (Le Guern 2003: 26). Par «univers de discours", nous pouvons entendre les termes utilisés et les caractères du concept pris en compte;

- l'objectif; etc.

\section{La dimension du concept}

Cette question du choix nous ramène à une autre notion, qu'on retrouve chez quelques terminologues, qui est celle de «dimension». On appelle "dimension» les critères de choix des caractères conceptuels retenus dans l'analyse d'un concept, donc le point de vue adopté dans une analyse quelconque. Et puisqu'il existe, comme nous venons de le voir, plusieurs critères qui conditionnent le choix des caractères, on peut en conclure qu'un concept peut avoir plusieurs dimensions, selon l'angle de vue envisagé. Depecker désigne ce fait par le nom de "pluridimensionnalité » (Depecker 2003: 85-86) d'un concept. 
Nous retrouvons la même notion chez Conceição désignée par le terme «multidimensionnalité» (Conceição 2005: 112).

Le concept //astéroïde// peut être ainsi abordé selon le critère de la composition de sa surface - riche en métal, en carbone, etc. -, ou celui de la zone de l'espace dans laquelle il se trouve - ceinture de Kuiper, ceinture jovio-martienne, points de Lagrange, etc.

L'analyse des dimensions d'un concept permet de le délimiter et de définir les relations qu'il entretient avec les autres concepts du domaine, donc de mieux structurer les connaissances à l'intérieur du domaine.

En effet, une fois les dimensions de chaque concept d'un domaine identifiées, il serait plus facile de catégoriser ou classifier ces concepts, et par la suite d'assigner à chaque concept son emplacement au sein du système de concepts formé par les relations conceptuelles du domaine.

Le concept //galaxie// peut être abordé sous la dimension de sa morphologie et peut ainsi générer cinq concepts spécifiques:

\begin{tabular}{|c|c|c|c|c|}
\hline & & galaxie & & \\
\hline $\begin{array}{l}\text { galaxie } \\
\text { spirale }\end{array}$ & \begin{tabular}{|c} 
galaxie \\
spirale barrée
\end{tabular} & $\begin{array}{l}\text { galaxie } \\
\text { elliptique }\end{array}$ & $\begin{array}{l}\text { galaxie } \\
\text { irrégulière }\end{array}$ & $\begin{array}{c}\text { galaxie } \\
\text { lenticulaire }\end{array}$ \\
\hline
\end{tabular}

Ou sous la dimension de son rayonnement électromagnétique et peut ainsi se subdiviser en deux concepts spécifiques:



De la même manière, on peut aborder le concept //planète// suivant ses propriétés physico-chimiques

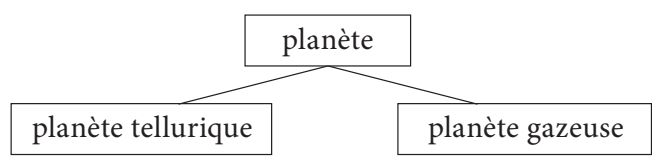

Ou suivant la position de son orbite par rapport à celui de la Terre:

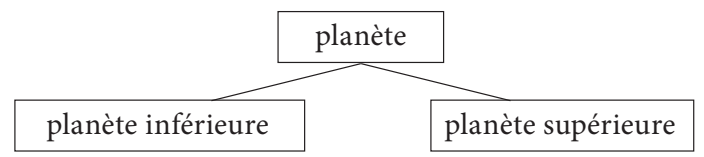

Ou suivant sa position par rapport au Soleil:

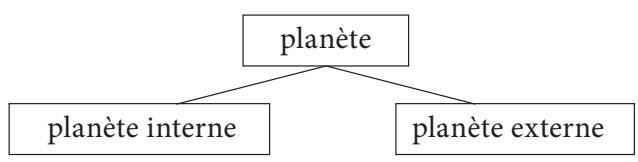


Cette structuration du système et la classification des concepts qui en découle permettront la catégorisation des objets auxquels réfèrent ces concepts.

Le concept //étoile// peut donc être abordé selon plusieurs angles, générant ainsi plusieurs classifications de l'objet «étoile».

//étoile//: //Objet céleste// constitué de //masse gazeuse très dense// //haute température// //émettant un rayonnement de lumière et de particules//.

$1^{\circ}$ Caractère pris en compte: //haute température// - Critère: la température $\rightarrow$ Classification de Harvard

\begin{tabular}{|l|l|l|l|}
\hline Classe & Température & Couleur & Raies d'absorption \\
\hline O & $30000-60000 \mathrm{~K}$ & bleue & azote, oxygène \\
\hline B & $10000-30000 \mathrm{~K}$ & bleue - blanche & hélium, hydrogène \\
\hline A & $7500-10000 \mathrm{~K}$ & blanche & hydrogène \\
\hline F & $6000-7500 \mathrm{~K}$ & jaune & métaux \\
\hline G & $5000-6000 \mathrm{~K}$ & jaune - orange & hydrogène, métaux \\
\hline K & $3500-5000 \mathrm{~K}$ & orange & métaux, oxyde de titane \\
\hline M & $2000-3500 \mathrm{~K}$ & rouge & métaux, oxyde de titane \\
\hline
\end{tabular}

$2^{\circ}$ Caractère pris en compte: //émettant un rayonnement de lumière et de particules// - Critère: la luminosité

$\rightarrow$ Classification de Yerkes

\begin{tabular}{|l|l|}
\hline Classe & description \\
\hline Ia & supergéantes très lumineuses \\
\hline Ib & supergéantes moins lumineuses \\
\hline II & géantes lumineuses \\
\hline III & géantes normales \\
\hline IV & sous-géantes \\
\hline V & naines \\
\hline VI & sous-naines \\
\hline VII & naines blanches \\
\hline
\end{tabular}

$3^{\circ}$ Caractères pris en compte: //haute température// //émettant un rayonnement de lumière et de particules// - Critères: la température et la luminosité

$\rightarrow$ Diagramme de Hertzsprung-Russell (CILF 2001 : 296)




Considérons les deux définitions suivantes de //Sirius// :

1. étoile la plus brillante du ciel.

2. étoile qui annonce les crues du Nil.

Il est évident que ces deux définitions ne datent pas de la même époque, la première étant contemporaine, la seconde remontant aux temps des Égyptiens anciens. N'empêche qu'elles définissent le même concept, à savoir le concept unique //Sirius//, et elles sont donc toutes deux valables. Valables mais pas commutables. En effet, le contexte, ou la situation, d'utilisation de ces définitions n'est pas le même. S'il s'agissait, par exemple, d'une étude portant sur le ciel chez les Égyptiens anciens, la seconde définition serait plus appropriée, alors que la première s'appliquerait mieux dans le cadre de la classification des étoiles les plus brillantes du ciel.

Donc la dimension envisagée dans ces deux définitions n'est pas la même, la direction de l'analyse étant elle aussi différente.

Passons maintenant à l'unité terminologique, influençable elle aussi par la dimension envisagée du concept nommé.

Pour rendre compte du concept comportant les caractères suivants: //étoile double// //composantes s'éclipsant mutuellement périodiquement// //variation de luminosité apparente//, il existe deux unités terminologiques différentes - «étoile binaire à éclipses » et "étoile variable à éclipses »-, chacune envisageant une dimension différente du concept.

Ainsi le terme «étoile binaire à éclipses» rend compte des éléments composant le système d'étoiles - //étoile double// // composantes s'éclipsant mutuellement périodiquement// -, alors que "étoile variable à éclipses» rend compte du mode de variation d'éclat - //variation de luminosité apparente// //composantes s'éclipsant mutuellement périodiquement//.

L'unité terminologique doit donc rendre compte d'au moins une dimension du concept, le choix de la dimension pouvant dépendre, comme on vient de le voir, de l'angle de vue sous lequel le concept est envisagé.

Cependant, le concept peut être appréhendé de différentes façons selon la langue du signe qui représente ce concept. En linguistique, Le Guern affirme que

Tous les traits de substance qui permettent de caractériser une classe d'objets ne se traduisent pas par autant de sèmes dans le lexique d'une langue donnée. Chaque langue opère un choix, et les sèmes constituant dans une langue le signifié du mot désignant habituellement une classe quelconque ne se retrouveront pas dans le signifié du mot par lequel une autre langue désigne la même classe d'objets (Le Guern 2003: 28)

les «traits de substance» étant les propriétés des objets et les «sèmes» les prédicats constitutifs des signifiés. Les propriétés des objets étant en relation directe avec les caractères des concepts, nous pouvons ensuite affirmer que les caractères qui permettent de caractériser un concept ne sont pas les mêmes pour toutes les langues.

Nous savons, en effet, que le découpage de la réalité diffère d'une langue à l'autre et d'une culture à l'autre. Ainsi le terme «éclipse» en français est utilisé pour désigner l'éclipse de Lune, l'éclipse de Soleil, ou l'éclipse de n'importe quel objet céleste, alors qu'en arabe, il n'existe pas d'équivalent exact de ce terme, la langue arabe faisant la distinction entre éclipse de Soleil traduit par «kousouf» et éclipse de Lune traduit par «khousouf». La catégorisation même de ces concepts est différente en français et en arabe. C'est que «les concepts ne sont pas nécessairement liés à des langues particulières. 
Ils sont cependant soumis à l'influence du contexte socioculturel qui conduit souvent à des catégorisations différentes» (ISO 1087-1 2000). Il y a donc la dimension socioculturelle qui influe sur le choix des caractères que traduit l'unité terminologique.

Ainsi le concept correspondant à: //zone////chromosphère solaire////regroupant////ensemble de facules// est exprimé en français par "plage faculaire», en mettant l'accent sur les caractères //regroupant// et //ensemble de facules// alors que la langue anglaise tient plutôt compte des caractères //zone// et //chromosphère solaire// avec le terme "chromospheric plage».

De même, le concept //lumière// //reflétée// //de la Terre// // et de son atmosphère// //éclairant///partie sombre// //du croissant de Lune// //couleur gris bleuté// est représenté en français par le terme « lumière cendrée» qui tient compte des caractères //lumière// et //couleur gris bleuté//, alors que le terme anglais «earthshine» met l'accent sur les caractères //lumière// //reflétée// et //de la Terre//.

Cette différence du choix des caractères apparaît également entre des langues aussi différentes que le français et l'anglais, d'une part, et l'arabe, d'autre part.

\begin{tabular}{|c|c|c|c|}
\hline concept & terme & langue & caractères pris en compte \\
\hline \multirow{3}{*}{$\begin{array}{l}\text { //explosion cataclysmique } \\
\text { d'une étoile qui peut pendant } \\
\text { un temps briller plus } \\
\text { vivement qu'une galaxie } \\
\text { entière de milliards d'étoiles// }\end{array}$} & supernova & Fr. & $\begin{array}{l}\text { //augmentation considérable } \\
\text { soudaine de luminosité// }\end{array}$ \\
\hline & supernova & Ang. & $\begin{array}{l}\text { //augmentation considérable } \\
\text { soudaine de luminosité// }\end{array}$ \\
\hline & 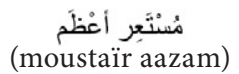 & Ar. & //explosion// //luminosité// \\
\hline
\end{tabular}

\begin{tabular}{|l|l|l|l|}
\hline concept & terme & langue & caractères pris en compte \\
\hline \multirow{3}{*}{$\begin{array}{l}\text { //instrument d'observation } \\
\text { qui permet d'augmenter la } \\
\text { taille apparente d'objet situé } \\
\text { au loin // }\end{array}$} & télescope & Fr. & $\begin{array}{l}\text { //instrument d'observation// // } \\
\text { augmenter la taille apparente } \\
\text { d'objet lointain// }\end{array}$ \\
\cline { 2 - 4 } & telescope & Ang. & $\begin{array}{l}\text { //instrument d'observation// // } \\
\text { augmenter la taille apparente } \\
\text { d'objet lointain// }\end{array}$ \\
\cline { 2 - 4 } & $\begin{array}{l}\text { d } \\
(\text { mirqab) }\end{array}$ & Ar. & //instrument d'observation// \\
\hline
\end{tabular}

\section{Conclusion}

À partir de l'analyse des caractères de plusieurs concepts, on a pu montrer l'importance de cette décomposition du concept qui nous a conduite à démontrer la «pluridimensionnalité» d'un concept, une pluridimensionnalité observée dans l'analyse des unités terminologiques, des arborescences et des définitions. Cette notion de "pluridimensionnalité», une fois établie, facilitera l'analyse des concepts et des systèmes de concepts en:

- clarifiant et simplifiant les relations qui existent entre les concepts d'un domaine;

- délimitant la circonscription de chaque concept au sein du système de concepts auquel il appartient;

- facilitant l’analyse des unités terminologiques;

- facilitant la rédaction de la définition;

- facilitant l'analyse comparative interlinguistique des concepts. 


\section{RÉFÉRENCES}

CILF (1989): Terminologie diachronique, actes du colloque organisé à Bruxelles les 25 et 26 mars 1988, Paris.

CILF (2001): Dictionnaire de Spatiologie, Sciences et techniques spatiales, Termes et définitions, Tome I, Paris, PUF.

Conceição, M. C. (2005): Concepts, termes et reformulations, Lyon, Presses Universitaires de Lyon.

DAYÉ, F. (1990): L’Encyclopédie des termes scientifiques arabes, Beyrouth, Dar al fikr al arabi et Damas, Dar al fikr.

Depecker, L. (2003) : Entre signe et concept, Paris, Presses Sorbonne Nouvelle.

Diderot, D. et J. D’Alembert (1750) : Encyclopédie ou Dictionnaire Raisonné des Sciences, des Arts et des Métiers, <http://portail.atilf.fr/encyclopédie/index.htm>.

Foucault, M. (1996): Les mots et les choses, coll. Tel, Éditions Gallimard.

Furetière, A. (1690): Dictionnaire Universel.

ISO (2000): Norme ISO 1087-1.

JouRdAK, M. (1950): L’Encyclopédie astronomique, Beyrouth, Librairie du Liban.

Le Guern, M. (2003): Les deux logiques du langage, Paris, Honoré Champion.

Thoiron, Ph., Arnaud, P., BÉjoint, H. et C. P. Boisson (1996): «Notion d'“archi-concept” et dénomination", Meta 40-4, pp. 512-524.

UAI, Union Astronomique Internationale, $<w w w . i a u . o r g>$.

Wikipedia, <www.wikipedia.org $>$. 